\title{
LA MEDIACIÓN DOCENTE EN LA COMPRENSIÓN DE LECTURA DE TEXTOS LITERARIOS
}

Silvia Méndez Ancbia

Resumen: Este articulo constituye una aproximación a un campo que ba sido poco estudiado en Costa Rica: el de la mediación docente en la comprensión lectora de textos literarios en el nivel de la enseñanza media. Pretende trazar algunas lineas de estudio sobre las estrategias que emplea una docente en particular al poner en contacto a sus estudiantes con los textos literarios. Es una investigación exploratoria, cualitativa, basada en el estudio de casos; de abi que no se pretende generalizar sus resuliados; todo lo contrario, se trata de plantear algunos temas que podrian servir de punto de partida para futuras investigaciones.

\section{Justificación}

\subsection{Un asunto cuyo estudio vale la pena}

¿Vale la pena estudiar el campo de la mediación docente en la lectura de textos literarios en la enseñanza media?

El discurso literario se ha considerado la expresión más alta de destreza lingüística. Rodino y Ross señalan que “...el máximo valor social en materia de expresión lingüística se asigna a la Literatura (en general, prescindiendo de diferencias de género, de épocas históricas, de escuelas o movimientos, o de estilo individual del autor)" (1997, p. 33). Tal valoración convierte este discurso en una fuente indispensable de conocimiento de las habilidades máximas del ser humano en el área de las destrezas lingüisticas y, por lo tanto, le confiere un uso ejemplificador.

Por su parte, la adolescencia -momento del desarrollo en que se hallan los estudiantes de enseñanza media- representa un periodo en que el joven empieza su tránsito hacia una etapa de operaciones formales ( $\mathrm{Pia}$ get, 1983) y a ello suele asociarse una mayor exigencia, por parte de este, sobre todo en el plano de las demandas académicas. Cuando hablo de nivel de exigencia, me refiero a las demandas de los alumnos en términos de que la educación sea atractiva e interesante, de modo que pueda competir con el atractivo de muchas otras situaciones que se les presentan, como los programas televisivos, los juegos de vídeo y la publicidad, entre otros. Mario Víquez, director del área de Niñez de la Defensoria de los Habitantes, señala como 
principales causas de la deserción de los estudiantes costarricenses, “...los problemas económicos y la falta de atractivo del sistema escolar frente a otras opciones para los jóvenes". (La Nación, 1999, la cursiva es nuestra.) Esta constituye una razón de peso para que el nivel de la enseñanza media se convierta en objeto de investigación.

Estando tales razones de por medio, iha respondido el ambiente intelectual costarricense a la preocupación por la enseñanza de la literatura en el nivel de la enseñanza media? Veamos.

\subsection{Del escaso interés por una problemática detectada}

Durante los últimos años se ha venido hablando de las dificultades que presentan los estudiantes en sus habilidades lectoras. Ese tipo de comentarios es frecuente en los medios de comunicación colectiva y en boca de los docentes. Puede observarse, como ejemplo, los argumentos que, para justificar su trabajo, dan las autoras de la serie "Despertando a las palabras":

"La inquietud que dio origen a esta propuesta surgió del enfrentamiento con una realidad inquietante y dolorosa: La gran mayoría de la población costarricense y una gran parte de los estudiantes de escuelas, colegios y universidades no gustan de la lectura y no saben leer." (Ducca y Rojas, 1993, p. 13).

Según información suministrada por María Emilce Rojas Salazar (1999), asesora nacional de Español del Ministerio de Educación Pública de Costa Rica, los ítemes de las pruebas de bachillerato en Español que presentan más resultados negativos son aquellos que se relacionan con habilidades interpretativas.

Existe, pues, la evidencia que confirma la opinión generalizada en cuanto al pobre desempeño lector de los alumnos de enseñanza media de Costa Rica.

Sin embargo, son escasas las investigaciones que atienden a esta problemática. Hay un marcado interés por investigar acerca de los procesos de lectoescritura en las fases iniciales del proceso escolar. Ese afán investigador va disminuyendo conforme avanzan los niveles de los alumnos: los estudios realizados en torno a la comprensión lectora suelen tomar como sujetos a estudiantes de primero y segundo ciclos; no es común que a los alumnos de enseñanza media se los considere sujetos de investigación.

También los lineamientos dirigidos a aplicar estrategias de lectura de textos literarios para estudiantes de enseñanza media, se han inclinado más por el análisis de las obras que por los procesos lectores propiamente tales. Así, es marcada la diferencia de tratamiento que se da en este punto según se trate de escolares o de colegiales.

En el caso de los primeros, las indicaciones para desarrollar las habilidades lectoras se suelen centrar en la motivación; por ejemplo, la metodología que apoya la enseñanza de la lectura se basa en estrategias de "animación", que implican no sólo la comprensión de lectura, sino también el goce y la reflexión. La serie "Despertando a las palabras" resulta un buen ejemplo de tal orientación; he aquí un fragmento tomado de los propósitos de dicha serie:

"Este trabajo se ha realizado pensando en destacar los rasgos fundamentales de la personalidad del niño: su vivacidad, su imaginación, su espontaneidad, su inteligencia, su creatividad, su capacidad de juego y de estremecerse frente a lo bello y frente a los sentimientos más nobles de la humanidad." (Ducca y Rojas, 1993, p. 14).

Siguiendo con el caso de los escolares, la mayoría de los libros de lectura dedicados a este nivel incorporan textos literarios breves, cuyo contenido llama la atención de los niños (sobre todo por su carácter fantástico); las actividades que plantean se dirigen a desarrollar la comprensión de lectura en niveles básicos y se acompañan de ejercicios de carácter lúdico.

Por su parte, los programas de estudio de Español del Ministerio de Educación Pública destinados al primero y segundo ciclos, son bastante "abiertos" en lo que se refiere al área de la lectura. Permiten que el docente escoja la mayoría de los textos. Además, las orientaciones que dan no son estrictas, sino 
que enfatizan en el desarrollo de los niveles de comprensión de lectura e incorporan pocos elementos de análisis literario propiamente tal.

Todo lo contrario sucede al llegar a la enseñanza media. En este nivel se contemplan poco las estrategias de comprensión de lectura. Por ejemplo, no hay manuales destinados específicamente a la "animación" de la lectura en los adolescentes, como sí los hay para niños en edad escolar. Lo que existen son libros de texto en que se presentan análisis de textos literarios, es decir, interpretaciones hechas por los redactores, y que los estudiantes leen para "comprender" mejor la obra literaria en cuestión. Los programas de estudio del Ministerio de Educación Pública, por su parte, están cargados de contenidos, con textos de lectura obligatoria, en cuya selección predomina el criterio de lectores adultos, y con estrategias de análisis que se orientan hacia la aplicación de conceptos procedentes de la teoría literaria y del estudio filológico. Véase el siguiente caso tomado del programa de estudios de Español de sétimo año; si bien en los principios generales de ese documento curricular se indica la necesidad de desarrollar una lectura creadora y crítica (Costa Rica, MEP, 1995, p. 11), los contenidos específicos se presentan en forma de categorías de estudio de corte descriptivo, las cuales por sí solas no implican el desarrollo de la comprensión lectora:

\section{“4.2. Lectura de textos literarios. \\ Leyenda, cuento y novela. \\ Análisis de los siguientes elementos:}

Narrador: tipos (omnisciente, testigo y protagonista); visiones (narrador > personajes, narrador < personajes, narrador $=$ personajes); organización secuencial de la historia narrada (lineal o perturbada); código apreciativo (valoración de las partes del mundo mostrado); tiempo. Mundo mostrado: espacios (físico, ético, religioso, juridico, educativo, económico, social, ecológico o psicológi$\mathrm{co}$ ); personajes (lo que dice ser y lo que hace); su interacción con los otros; su código apreciativo (valoración del mundo mostrado).

Relaciones del texto con el contexto sociocultural, con el género literario y, en el caso de la novela María, con el movimiento romántico." (Costa Rica, MEP, 1995, p. 19).

Puede verse, entonces, que la problemática del pobre desempeño de los estudiantes costarricenses de enseñanza media en la comprensión de textos literarios, es un asunto que ya se ha detectado. Sin embargo, ante tal situación, ha sido escaso el interés por investigar este campo de estudio y por implementar estrategias específicas que consideren las necesidades del desarrollo de los adolescentes, como sí parece haberse hecho en el caso de los escolares. Son muy pocas las investigaciones que se plantean como objeto de estudio la comprensión lectora de estudiantes de enseñanza media, a la vez que los modelos de acercamiento a los textos que se desprenden del programa de estudios del Ministerio de Educación Pública se centran más en el análisis a partir de categorias específicas, que en el desarrollo de la comprensión lectora propiamente tal.

\subsection{Preguntas sin respuesta}

Vista dicha situación, me formulo varias interrogantes:

- $\quad$ ¿Se considerará, acaso, que los procesos de comprensión lectora, como tema de investigación, se agotan en las etapas iniciales de la lectoescritura? Si fuera así, se estarían dejando de lado dos factores importantes: que la capacidad para elaborar inferencias -elemento básico para la comprensión de los textos literarios- se desarrolla mejor a partir de cierta edad -se ha notado una mayor habilidad en este aspecto cuanto mayor es la edad de la persona (Carrasco, 1999; Riffo, 1997)-; y que el conocimiento previo, otro elemento que se afina con el paso del tiempo, es más rico en los estudiantes conforme avanzan en edad y en escolaridad. De tal manera, los alumnos de enseñanza media constituyen, por sus características etarias, un grupo particular para el estudio de la comprensión lectora, dado que sus habilidades inferenciales y su conocimiento previo marcan una diferencia respecto de los niños en edad escolar.

- $\quad$ Es posible que se crea que conviene motivar más a los escolares que a los 
colegiales debido a que aquellos necesitan más despertar su interés por la lectura que estos? Si privara tal criterio, se estaría desestimando el hecho de que los adolescentes se hallan en un periodo en que buscan definir un plan de vida y que, en este, una de las funciones docentes es motivar al estudiante de una manera particular: problematizando (Krauskopf, 1993).

- ¿Se pensará, tal vez, que los estudiantes de enseñanza media ya han desarrollado habilidades lectoras tales que llevan a implementar programas en que predomina un abordaje teórico e historiográfico de la literatura, en detrimento de las destrezas de lectura? Si planteáramos de tal manera el asunto, estaríamos obviando el pobre desempeño en la lectura en general por parte de colegiales -e incluso de estudiantes universitarios- que ha sido detectado en algunas investigaciones.

- La escasez de investigaciones en el campo de la enseñanza de la literatura en enseñanza media, ¿podría considerarse un reflejo de la situación de crisis y abandono que atraviesa el sistema educativo costarricense, la cual se acentúa en los niveles correspondientes al tercer ciclo de la Enseñanza General Básica y el ciclo de Educación Diversificada? Según el Informe de Desarrollo Humano del Programa de las Naciones Unidas para el Desarrollo (PNUD), la tasa de cobertura de la enseñanza media es de $55,8 \%$, frente a un $95,1 \%$ en primaria. En cuanto a la tasa de deserción en enseñanza media, ese documento arroja las siguientes cifras: $10,5 \%$ en la educación académica diurna, $12,2 \%$ en la técnica y un $36,6 \%$ en la nocturna; frente a un 4,9\% en la educación primaria (La Nación, 1999).

\subsection{Un problema justificado}

Recapitulemos. La población de estudiantes de enseñanza media presenta una situación particular: están en una edad en que se puede dar un mayor aprovechamiento de ciertos procesos (los de lectura inferencial y el manejo de conocimientos previos); además, en esta etapa de la vida la motivación sigue siendo necesaria y adopta una variante: la problematización. Pese a contar con estos elementos en su favor, esta población presenta grandes dificultades para resolver satisfactoriamente aquellos itemes de las pruebas nacionales relacionados con la destreza de lectura interpretativa; además muestran índices de deserción muy altos en lo que a educación formal se refiere.

Por tales motivos, y ante la escasez de investigaciones en este campo, considero necesario que se realicen estudios acerca de la lectura literaria en el nivel de la enseñanza media. En este artículo investigo las estrategias que emplea una docente de San José de Costa Rica para desarrollar procesos de comprensión de lectura de textos literarios en sus estudiantes, así como las dificultades que enfrenta en esta tarea. Con tal objeto, he delimitado el siguiente problema: ¿Cuáles estrategias de comprensión de lectura de textos literarios promueve una profesora de Español de enseñanza media en sus estudiantes durante las actividades de clase y cuáles dificultades enfrenta en esa tarea?

\subsection{Mi intención}

Por tratarse de un estudio exploratorio, este constituye una aproximación primera a la problemática de los procesos de comprensión lectora de textos literarios y a la participación del docente como mediador en tales procesos. No se pretende, por lo tanto, generalizar sus resultados; todo lo contrario, plantea algunos temas que podrían servir de punto de partida para otras investigaciones; de modo que funcione como trabajo pionero para aquellos estudiosos del área de la enseñanza de la literatura que deseen incursionar en este campo virgen.

\section{Una aproximación bibliográfica}

Esta aproximación bibliográfica comprende dos momentos: la presentación de algunas posiciones teóricas acerca de la lectura y 
la referencia a ciertas características de los textos llamados "literarios". Ello en un afán por construir un marco teórico provisional que sustente el análisis de la práctica docente en la enseñanza de la lectura de textos literarios.

\subsection{Texto y lector, lector y texto}

La comprensión de lectura implica la presencia de un texto y un lector. Existen diversas posiciones teóricas al respecto. La primera de ellas se centra en el contenido del texto y considera el acto lector como la habilidad de "recuperar" lo que ha escrito el autor. La segunda corriente enfatiza en el lector y concibe el acto de la lectura como la actualización de los esquemas de este al entrar en contacto con el texto. La tercera postura, denominada "transaccional", afirma que intervienen tanto el texto como los esquemas del individuo, de manera tal que cada uno condiciona y es condicionado por el otro. (Carrasco, 1999).

Viendo el asunto de la comprensión de lectura desde una óptica transaccional, Isabel Solé (1996) habla de tres requisitos o condiciones para el proceso de la comprensión. El primero de ellos radica en el texto: la claridad o coherencia de sus contenidos, su estructura (que sea conocida para el lector), el léxico, la sintaxis y la cohesión interna. Otro requisito tiene que ver con el lector: este debe poseer cierto conocimiento previo que lo faculte para comprender el contenido del texto. La tercera condición también se asocia con el sujeto que lee: supone la utilización de estrategias diferentes según la intención de la lectura (aprendizaje, información, entretenimiento...).

"Comprendemos -afirma Solé (1996)- porque podemos establecer relaciones significativas entre lo que ya sabemos, hemos vivido o experimentado y lo que el texto nos aporta. Si comprendemos lo que está escrito es porque podemos ir relacionándolo con cosas que ya conocíamos e ir integrando la información nueva en nuestros esquemas previos..."

Además, la estructura del texto se plantea como un requisito por considerar en la comprensión de lectura. Pujol y Vivas (1997) señalan que la forma en que se encuentra estructu- rado un texto influye en su comprensión por parte del lector; esto se debe a que el lector posee esquemas mentales previos que le ayudan a relacionar los contenidos y la estructura de los textos con el fin de comprender el mensaje; así, diferentes tipos de texto activan distintos procesos cognitivos. Bendito (1989) sostiene que una estrategia para mejorar las habilidades de comprensión lectora, radica en el conocimiento y práctica de determinadas estructuras textuales.

Ahora bien, aquí se ha hablado de la lectura en general. Pero, ¿qué sucede con la lectura de textos literarios? En los párrafos que siguen, defino algunos puntos acerca de la literatura e intento conectarlos con algunos de los aspectos aquí señalados.

\subsection{Habia una vez unos textos llama- dos "literarios"...}

Los textos conocidos como "literatura" forman un discurso con unas características particulares, si se los compara con otro tipo de textos como los científicos, los periodísticos y los jurídicos, por citar solo unos cuantos casos.

En teoría literaria, es materia de nunca acabar la discusión acerca de qué es la literatura. Por tal motivo, he decidido partir de los rasgos del lenguaje literario que proponen Rojas, Víquez y Sánchez (1996) en el libro de texto Español 8 de la serie "Hacia el siglo XXI", por considerarlo una fuente autorizada $y$, además, porque tal caracterización está planteada para estudiantes de enseñanza media. Los rasgos que definen el lenguaje literario -y que lo dotan de una estructura particular, que debe conocer el lector a la hora de entrar en contacto con este para su comprensión-son estos:

- Pluralidad: El texto literario se puede interpretar de muchas formas.

- Connotación: El texto literario obtiene su pluralidad de la connotación, es decir, las palabras que lo componen tienen, además de un significado denotativo, un significado connotativo, que toma su valor de la cultura de la cual el texto forma parte. 
- Ficcionalidad: El texto literario se refiere a un mundo creado a partir de sus propias palabras, no a un mundo real. Adquiere este carácter por el contexto en que se da; debe darse en un contexto literario.

- Dialogismo: El texto literario se halla siempre en relación con los otros textos y con los lectores: "...el lector lleva consigo, a la hora de interpretar el texto literario, una serie de otros textos que recuerda y le sirven para interpretar el nuevo texto que está leyendo". (Rojas y otros, 1996, p. 29).

- Intertextualidad: Cada nuevo texto literario se genera gracias a un diálogo con otros textos que han existido previamente. (Aquí el término "textos" se utiliza en un sentido amplio, para incluir no sólo los escritos, sino también otros discursos culturales).

Además de esos rasgos generales que puede atribuírsele a los materiales escritos considerados como literarios, existen otros rasgos que diferencian estos textos entre sí. Por ejemplo, la distinción según el género: novela, cuento, poesía, drama y ensayo (y dentro de esta, otras subdivisiones posibles, como la que se daría entre novela policiaca, novela sentimental, etc.). Asimismo, la distinción de acuerdo con el movimiento literario en el cual se inscriban: Modernismo, Realismo, Romanticismo, Naturalismo, Neoclasicismo, Vanguardismo...

Todos estos elementos suponen una competencia particular del lector. En la comprensión de lectura de textos literarios influyen, por lo tanto, los aspectos propios de cada texto en particular (sus características que lo hacen tal, así como el género y el movimiento literarios). Algunos de esos aspectos -como el género y el movimiento literarios- deben formar parte del conocimiento previo del lector, de modo que le aseguren activar los esquemas necesarios para la comprensión de lectura. Otros, en cambio, suponen competencias que se acti- van al contacto con el texto -como el dialogismo, la pluralidad y la connotación-.

\subsection{Y las posibilidades quedan abiertas}

El campo de la lectura es muy amplio. En él se conjugan elementos de la más diversa índole, que involucran tanto al texto en sí como al sujeto lector que entra en contacto con él para construir su significado.

Si consideramos la lectura de textos literarios, el panorama, lejos de delimitarse, se torna más complejo aún, pues intervienen las características propias de este discurso (ficcionalidad, pluralidad, connotación, dialogismo e intertextualidad) junto a otros elementos formales (como el movimiento literario y el género).

\section{Metodología}

\subsection{Poniendo entre paréntesis (El paradigma naturalista)}

He encuadrado esta investigación dentro del paradigma naturalista, según lo definen Dobles, Zúñiga y García (1996).

A continuación detallo las premisas básicas de este paradigma. En términos ontológicos, la realidad es una construcción social y no constituye, por tanto, un todo homogéneo. Epistemológicamente hablando, el sujeto y el objeto del conocimiento interactúan y son inseparables; la principal implicación de esta premisa radica en el enriquecimiento de las posibilidades de interpretación de la realidad. Una de las premisas heurísticas de esta investigación es la de que los resultados obtenidos no son susceptibles de generalización, pues las experiencias con que entré en contacto se hallan determinadas por ciertas condiciones específicas; más que la generalización, me interesó determinar las variaciones que adoptaba la mediación docente en la lectura de textos literarios al interior de una situación particular. Por último, al paradigma naturalista se asocian valores tales como la necesaria interrelación entre el sujeto y el objeto de la investigación; por tal motivo, si bien la persona que 
investiga debe manejar una posición teórica definida, esta ha de ser lo suficientemente flexible como para considerar las posibles variaciones que, en este marco de referencia, puedan darse a partir de la información suministrada por la persona sujeto de estudio.

\subsection{Tipo de investigación}

Esta investigación es exploratoria, basada en fuentes primarias y cualitativa. Según Sierra Bravo (1994), las investigaciones exploratorias no pretenden medir variables ni estudiar las relaciones de influencia entre ellas, sino plantear un primer acercamiento a un problema. Los datos primarios son aquellos tomados de primera mano: en este caso, se trató de un testimonio y una entrevista a una docente. La metodología cualitativa se define, según Taylor y Bogdan (1992), como aquella que genera datos descriptivos, es decir, las propias palabras de la gente, ya sea en forma oral o escrita, y la conducta observable.

Además, el presente trabajo adopta la forma de un estudio de casos. De acuerdo con Stake (1998), este se caracteriza por ser cualitativo y naturalista, a la vez que constituye una base pobre para la generalización, o sea, que solo se puede hacer generalizaciones menores (aplicaclas al sujeto en estudio); más bien, su cometido es la particularización. Como supone un estudio a profundidad de uno o varios sujetos, su énfasis está puesto en la interpretación. Su objetivo no es el comprobar una hipótesis, sino la comprensión del sujeto en su totalidad. En esta investigación apliqué el denominado "estudio instrumental de casos": aquel que se orienta a comprender una situación, y no tanto a entender a la persona seleccionada como objeto de estudio.

\subsection{Método}

El método empleado tomó, como unidad de investigación, la forma como realiza la mediación pedagógica en la lectura de textos literarios, una profesora de Español de enseñanza media, quien labora en un colegio privado de
San José de Costa Rica. Aquí me referiré a ella como $M$

El trabajo de campo se compuso de dos fases: una primera fase destinada a obtener datos de la experiencia de la profesora de Espanol al enseñar literatura, y cuyo instrumento fue el testimonio; y una segunda fase, en la cual recurrí a la entrevista a profundidad.

Para procesar la información empleé la técnica del análisis de contenido. La materia prima a partir de la cual apliqué esta técnica la constituyeron el testimonio escrito de la docente, así como los datos tomados de la entrevista a profundidad.

Los resultados provenientes de la aplicación de esta técnica de análisis a los datos obtenidos del testimonio y la entrevista, los presento en los siguientes apartados. Allí aparecen agrupados en grandes categorias, las cuales a su vez se subdividen en categorias más específicas. Al mismo tiempo, incluye la reflexión conceptual, presentada a manera de diálogo entre los resultados y algunas de las premisas teóricas que sustentan el apartado de "Aproximación bibliográfica".

\section{Enseñar a leer literatura: una empresa difícil}

\subsection{Dificultades en la enseñanza de la literatura}

De las categorias que definí para realizar el análisis de contenido, la que más se repite es la denominada "dificultades", que incluye obstáculos de diversa índole que $M$. señala al referirse a su quehacer en la enseñanza de la literatura. De modo que esta constituye una categoría amplia, la cual abarca orros subcomponentes que defino a continuación.

$M$. alucle a varias dificultades que enfrenta en su labor de fomentar la comprensión lectora de textos literarios en sus alumnos de enseñanza media. Una de ellas tiene que ver con los textos, específicamente se refiere a la selección de textos literarios hecha en los programas de Español del Ministerio de Educación 
Pública. Otra se relaciona con una posible metodologia o estrategia de lectura: el análisis literario indicado en tales programas. Otra dificultad detectada se enfoca hacia la formación docente. El lector, en este caso el estudiante, constituye otro punto de reflexión, pues presenta dificultades en cuanto a motivación y conocimiento previo. Y la última se relaciona con el carácter propio de la disciplina literaria.

\subsubsection{Los textos: la selección de textos literarios en los programas oficiales}

El programa de estudios de Español de enseñanza media diseñado por el Ministerio de Educación Pública, es decir, el programa oficial, dispone una serie de pautas en relación con la lectura de textos literarios. Una de ellas consiste en un listado de obras, cuya lectura es de carácter obligatorio; de estas, algunas son evaluadas en las pruebas nacionales de noveno año y de bachillerato (en este último caso, todas las obras estudiadas en décimo y undécimo años forman parte del temario de examen). En el recuadro siguiente pongo un ejemplo de los textos de lectura obligatoria: los correspondientes a décimo año. He incluido datos como la nacionalidad del autor y el siglo a que pertenecen, ya que estos elementos son comentados por $\mathrm{M}$.

\section{Textos de lectura obligatoria en décimo año}

Epopeya: La Odisea, de Homero (Antigüedad griega). Novela: Don Quijote de la Mancha, de Miguel de Cervantes (España, s. XVII). Viaje al reino de los deseos, de Rafael Ángel Herra (Costa Rica, s. XX).

Cuento: "La sequía", de Carlos Salazar Herrera (Costa Rica, s. XX). "Clarisa", de Isabel Allende (Chile, s. $\mathrm{XX}$ ). "La casa de Asterión", de Jorge Luis Borges (Argentina, $s, \mathrm{XX}$ ).

Lírica: "Oda a Afrodita", de Safo (Antigüedad griega). "Los heraldos negros", de César Vallejo (l'erú, s. XX). "Un carnívoro cuchillo", de Miguel IIrmáncice (España, s. XX). "Penélope", de Nur Ropls (Cota Rica,s. $\mathrm{XX}$ ).

Drama: Prometeo Encichnato,

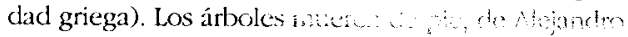

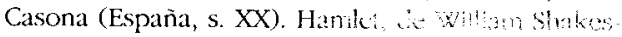
peare (Inglaterra, s. XVII).

Ensayo: "Abel y Caín en el ser hrinco di la ación costarricense", de Abelardo Bonilla (Costa Rica, s. XX).
Además de pertenecer a épocas y lugares diferentes, estos textos representan corrientes literarias y visiones de mundo muy diversas, incluso en los casos en que su producción data de la misma centuria. Por ejemplo, La sequia es un texto realista, en tanto que Viaje al reino de los deseos es literatura maravillosa.

M. considera que la selección de textos literarios de los programas es "pésima". Los factores que motivan esta calificación son los siguientes:

- Falta de adaptación al nivel comprensivo de los estudiantes: Los temas no son del interés de ellos y el léxico en algunos casos resulta incomprensible. Los textos literarios de los programas de décimo y undécimo no se adaptan -dice M- a estos chiquitos. Es una literatura suicida. (En cursiva aparecerán todas las citas textuales tomadas del testimonio y de la entrevista de M.) $Y$ añade que en esos textos aparecen metáforas muy "elevadas". Para ejemplificar el carácter "suicida" de tales obras y el lenguaje dificil que emplean, cita los casos de "Los heraldos negros", de César Vallejo, y de "Un carnivoro cuchillo", de Miguel Hernández. M. afirma que una posible causa de esa falta de adaptación radica en que los redactores de los programas no consideran a los estudiantes a la hora de seleccionar los textos.

- Falta de secuencia cronológica de los textos seleccionados. Como se puede observar en el recuadro que ilustra los textos de lectura obligatoria para décimo año, se pasa de la Antigüedad clásica al siglo XX y se devuelve luego al Renacimiento. Al respecto, dice M.: Usted no puede ubicarse adecuadrmenle, si los anitores som escogidos sin una se. cuencia bistónica-cronológica que onoyz si ponencia. Esta carencia de un hilo cronológico tiene varios efectos; según M., no favorece la moivación de los estudiantes ni la compration: Como los programas presenton la lieratura por pedacitos, los muchacbos no pueden involucrarse porque les toca bacer un análisis fragmentario. 
- Incomprensibilidad de algunos textos. Para M., la incomprensibilidad de algunos textos es fuente de dificultades debido a que la preparación de los docentes en las universidades no asegura que ellos puedan manejar ese tipo de textos.

Estos tres aspectos a que alude M. y que he ubicado bajo el.rubro de "dificultades por la selección de los textos literarios", corresponden a uno de los elementos del proceso de comprensión lectora, a saber, el texto. Parto aquí de los postulados de la teoría de la transacción, formulada por Louise Rosenblat, quien sostiene que, en el acto de la lectura, el lector "transactúa" con el texto, y también con el medio cultural, social y personal, de modo que "El significado no reside ya hecho en el texto o en el lector, sino que sucede durante la transacción entre el lector y el texto" (citado por Dubois, 1995, p. 8).

Con la aclaración anterior quiero destacar que, para efectos de la discusión de resultados de esta investigación, el texto literario es solo uno de los elementos que forman parte de la comprensión lectora. No es el más importante, pues entran en juego otros elementos, como el estudiante, el docente y las estrategias de lectura. Tales elementos los analizaré más adelante.

\subsubsection{Una posible estrategia de lectura: el análisis literario propuesto en los programas oficiales}

Como señalé en la justificación, los programas oficiales que rigen la enseñanza de la literatura en Costa Rica, presentan un antisis litario que concmple une serix do chmonos simiarcs para todas las obras literarias que se incluyen como lecturas obligatorias. Para mayor claridad, sugiero remitirse al apartado 1.2, donde aparece un ejemplo tomado del programa oficial correspondiente a sétimo año.

Según M., en este aspecto los programas están "mal elaborados". El análisis que proponen se caracteriza por ser esquemático y homogeneizante: ...parecieran baberlos becho en una plantilla igual para todas las literaturas: autor, yo lirico, contexto, tipo de narrador; la docente critica esta predeterminación de categorias, incapaces-escribe ella-de abarcar la esencia de una obra...

Este tipo de aproximación (fragmentaria) a los textos literarios genera una reacción de rechazo por parte de los estudiantes. El porqué del fallo de esta propuesta de lectura se ve -siguiendo con lo planteado por $\mathrm{M}$ - en cuatro factores: a) no considera a los estudiantes, b) no toma en cuenta a los profesores, c) no se adapta a la realidad y d) su carácter es experimental. Estas son las palabras de la docente: Pareciera que los muchachos y profesores labriegos sencillos, sólo somos conejillos de Indias, nada más.

En cuanto a los procesos de comprensión lectora, estas categorías no ayudan a comprender los textos. A criterio de M., son innecesarias, ya que el estudiante puede llegar a una interpretación sin ellas, o sea, tomándolas en cuenta, pero sin que se le pidan como tales. Por tal motivo, este sistema no enseña a interpretar. Respecto de la ineficacia de esta forma de analizar todos y cada uno de los textos, la docente señala: ¿Cómo les va a preguntar uno sobre el tipo de narrador en el Quijote, cuando no bay uno, sino un montón: no se sabe si es Cide Hamete Benengeli, o quién?

Según el criterio de M., el análisis de los textos literarios que propone el Ministerio de Educación Pública no resulta válido como estrategia de comprensión de lectura para estudiantes de enseñanza media.

\subsubsection{La mediación pedagógica y la formación docente}

Otro tipo de dificultades radica en la formación docente de los profesores de Español. M. la califica de "didactismo" y la acusa de hacer que los estudiantes pierdan la capacidad de abstracción, de formular pensamiento.

¿Cómo se caracteriza ese "didactismo"? En palabras de M., es convertir la técnica didáctica (se refiere a carteles, juegos, etc.) en un objetivo, dejando de lado el contenido mismo. Se asocian a esta práctica actividades inflexibles; así, por ejemplo, la organización de la 
lección sé basa en partes cuyos límites temporales son estrictos. Nótese la crítica a esta forma de impartir lecciones en el siguiente comentario: En la clase siguiente, se aclaran dudas un ratito tho marcado por el reloj, como aconsejân "los grandes didactistas, sino basta donde se considere necesario aunque me brinque todo el prógrama del día, lo más importante es que no queden dudas.

De acuerdo con M., en la formación que ella ha recibido, el acto docente se centra en la técnica. Hay un interés por lo formal, no por que las técnicas se articulen con los contenidos. La técnica es el objetivo -señala, y continúa-, para ellos es preferible dar las técnicas que dar la clase. Sólo nos enseñan lo formal -dice en otro momento-, nada de contenidos.

Acerca de las observaciones de $\mathrm{M}$. respecto de su formación docente, voy a comentar dos puntos. El primero estriba en la inexactitud de criterios para distinguir lo que corresponde al campo de la didáctica. parece haber una confusión entre técnicas e instrumentos.

Es evidente que la profesora maneja una distinción entre fondo y forma, o entre contenido y técnica. A su preparación como docente corresponderian los aspectos formales, de técnicas; y a su formación filológica, los de fondo o de contenido.

En estos comentarios de $M$. encuentro resabios, en lo que a historia de la formación docente en Costa Rica se refiere, de la llamada "lucha entre el qué y el cómo", que entablaran, a finales de los años cincuenta, la Facuiltad de Ciencias y Letras y la Facultad de Educación, ambas de la Universidad de Costa Rica. La primera abogaba por que se volviera a un modelo anterior, en el cual los profesores de ensertanza media recibían una formaçión unitaria", a cargo de la unidad académica en que se especializaban, y con unos arantos cursos de educación impartidos por esa misma unidad académica. La Facultad de Educación defendía, por su parte, el modelo instaurad8 pocós años antes -llamado por Maúa Eugenia Dengo, el "modelo de la segunda etapa"-; er el cual la formación de do- centes de enseñanza media estaba a cargo, en forma conjunta, de los departamentos de la Facultad de Ciencias y Letras y de la Facultad de Educación. Este último modelo, instaurado desde 1955 es el que, con algunas modificaciones, se mantiene vigente en la actualidad. (Dengo, en prensa).

Volviendo al caso de M., pero sin dejar de lado ese enfrentamiento entre "el qué y el cómo", no parece que haya una articulación entre la docencia y el saber filológico en términos de aportar técnicas o instrumentos didácticos específicos para atender a las habilidades propias de los procesos lectores (si nos referimos al campo de la literatura). Esta, que es solo una suposición basada en un caso específico, parece verse respaldada por la escasez de investigaciones hechas en Costa Rica sobre enseñanza de la literatura en el tercer ciclo de la Enseñanza General Básica y en el ciclo de Educación Diversificada.

Con este punto de la formación docente, entro en un campo que va más allá del acto lector, en que intervienen, como dije en un apartado anterior, el texto, el lector y su contexto, en una verdadera transacción. Aquí he incorporado otro elemento, el docente, quien tiene a su cargo la mediación pedagógica.

\subsubsection{De los lectores: los estudiantes de enseñanza media, su desinterés y la falta de contexto para sus lecturas}

El análisis esquemático de los textos literarios produce una respuesta de rechazo en los estudiantes. Todo lo encasillan borrorosamente -escribe M. en su testimonio-, baciendo de la acción literaria una verdadera aberración, casi un castigo, que es como lo sienten los muchachos. En otra parte apunta: ...he ballado casi exactamente lo mismo: desidia, rechazo, casi odio de los muchacbos cuando se les pronuncia tal palabra (se refiere a la "literatura").

Las causas de ese desinterés son, además del análisis fragmentario de los textos, la obligatoriedad de estos y el que su lectura se supedite a una prueba. En este punto intervienen, entonces, tanto la actitud de los estudiantes como 
las disposiciones de las autoridades ministeriales. Han sido obligados, amenazados, a tragarse textos que para ellos no significan absolutamente más que una molestia y un examen, en donde deben contestar unas preguntas y olvidarse del asunto, escribe $M$.

Llegando a este punto, encuentro dos elementos en que conviene introducir el aporte de la teoría literaria y de la literatura misma.

En una de sus frases geniales, Borges (1988) dice que "...la idea de la lectura obligatoria es una idea absurda: tanto valdría hablar de felicidad obligatoria" (p. 107). Si se imponen textos literarios a los estudiantes, es difícil que ellos los encuentren placenteros; de ahí la dificultad que les plantea, a los docentes, el fomentar la lectura de dichos materiales.

Para uno de los más importantes teóricos de la literatura, el francés Roland Barthes, el placer del texto tiene que ver con las posibilidades subversivas de la palabra. Dicha subversión del discurso dominante se logra mediante la pluralidad de significados del texto literario. En la pluralidad, la ideología, aunque continúa existiendo, se halla burlada, pues el lector puede resistir su contagio: muchas voces confluyen como para que la ideología imponga dictados unilaterales.

Unos textos literarios que se plantean como obligatorios y cuya "comprensión de lectura" se mide en unas pruebas nacionales, constituyen claros ejemplos de fuentes de displacer, de desmotivación y de negación de la pluralidad subversiva de la literatura.

Paso, ahora, a otro punto relacionado con los estudiantes, o sea, con los lectores en este caso. Según M., ellos carecen de la información contextual necesaria para comprender los textos literarios; dice que Una dificultad para enseñar esta literatura es que los mucbachos no tienen el minimo contexto. Además, no comprenden la literatura porque no poseen una idea de la secuencia que ella implica; sin embargo, cuando sí disponen de alguna información contextual, esta la componen ideas deformadas de la realidad, propias de una enseñanza tradicional y de la influencia de la ideología dominante. (Cuando los mucbachos tienen ideas del con- texto, por lo general estas son erradas, deformadas, como en el caso de la llegada de los españoles a América, donde demuestran tener ideas tomadas de la Cartilla de Fernández Guardia).

Aquí M. introduce otro elemento vital para la comprensión lectora, si la consideramos desde de la teoría de la transacción: el conocimiento previo. Para Isabel Solé, una condición de la comprensión de lectura tiene que ver con el conocimiento previo del lector: este ha de contar con la información necesaria para comprender el contenido. Refiriéndose a esta condición, la comprensión de la lectura dependerá "...de la posibilidad de que el lector posea un bagaje que le permita abordar el texto, o bien, si no posee esos conocimientos, que se le ofrezca la ayuda que necesita, es decir, que se le brinden, que se le guíe." (Solé, 1996, p. 12).

Para M., los muchachos de enseñanza media no poseen un conocimiento previo que les permita comprender los textos literarios: ... no saben nada, no saben cómoù ubicar.

Debe observarse que las teorías da ta lectura que sirven de susterto para esta discusión de resultados, no tratan éspecificamente el campo de la lectura literaria. En este campo, el asunto del conocimiento previo se torna aún más difícil de mánejar porque el lenguaje literario es ficcional, es decir, crea realidades por medio de las palabras tomando como base referentes reales. Por ello, el conocimiento previo incluye, según mi criterio, aspectos de ubicación histórica, filosófica y de movimiento literario, entre otros.

\subsubsection{Un saber $\sin$ normativa: la literatura}

La última de las dificultades se relaciona con la disciplina literaria.

M. encuentra difícil enseñar literațura porque esta no se halla normada, no tiene técnicas preestablecidas para su enseñanza, como tampoco una secuencia lógica que haga estrictamente necesario dar un paso antes de otro. Según ella, todo lo contrario sucede con la gramática, que está normada, posee técnicas establecidas que facilitan su enseñanza (como los mapas conceptuales) 
y presenta una secuencia lógica para su exposición; por ejemplo, no se puede enseñar la oración transitiva sin haber visto antes el complemento directo. Por eso, afirma que es más fácil enseñar gramática que literatura.

\subsection{Estrategias para fomentar la comprensión de lectura de textos literarios desde la mediación docente}

Las estrategias que se desprenden del discurso de $M$. enfatizan en la motivación, la ubicación contextual y el reconocimiento de la individualidad de cada estudiante. Además, se basan en una determinada concepción de la literatura y de la función u objetivo de la enseñanza de la lectura literaria.

\subsubsection{Aspectos generales: noción de literatura y objetivo de la enseñanza de la lectura literaria}

M. sostiene que la literatura es problematizadora, es decir, que no presenta la realidad tal y como lo han hecho siempre, sino que destaca lo diferente. Cita como ejemplo el caso de la novela Asalto al paraíso, de Tatiana Lobo, donde se habla de la conquista de América en términos del saqueo y la explotación que realizaron los españoles.

Desde mi punto de vista, esta forma de ver la literatura se relaciona con una estrategia que Dina Krauskopf (1993) recomienda a los profesores que tratan con adolescentes: la problematización. Esta actitud implica que el profesor reconozca en esta etapa de la vida un momento por sí mismo, y no solo como un paso hacia la vida adulta. Si adopta esta posición, el docente dejará a un lado la estructura verticalista profesor-alumno, y podrá empezar a intercambiar conocimientos con los estudiantes.

Por otra parte, M. plantea que la literatura implica también una actividad: interpretar. Con esta idea, aparece una noción de literatura que lleva implícito al sujeto lector, pues sin este no habría interpretación y, por lo tanto, tampoco significado alguno. De este comentario de $M$. puede desprenderse una posición transaccional de la comprensión lectora.

El objetivo de la lectura literaria se relaciona con tres elementos: el desarrollo de la creatividad (Eso es lo que quiero que saquen de lo literario, su propia creatividad, su propia inquietud), la creación de un pensamiento abstracto y el logro de una formación integral ( $\mathrm{La}$ literatura se enseña para que el estudiante aprenda a integrar los aspectos de su vida).

Como puede verse, estos objetivos apuntan tanto hacia aspectos del desarrollo cognoscitivo (operaciones formales), como del autoconocimiento (inquietudes propias, integración de aspectos vitales).

Estas dos dimensiones tienen relevancia en el desarrollo del adolescente. Así, el pensamiento operacional formal puede proveer de herramientas importantes para la interpretación literaria y, a su vez, esta puede contribuir a estimular tal tipo de operaciones. Estas son algunas características del pensamiento operacional formal citadas por Craig (1997): capacidad de abstracción y habilidad para la especulación, con libertad respecto del medio y sus circunstancias; capacidad para formular, probar y valorar hipótesis; habilidad para anticipar; desarrollo de procesos de segundo orden, que implican la reflexión sobre los propios pensamientos.

También le corresponde al periodo de la adolescencia una tarea delicada, como es la formación de la identidad. Son múltiples las fuentes a partir de las cuales el joven va formando una identidad propia: la familia, los amigos del barrio, los compañeros del colegio, los modelos de la televisión, entre otros. Los personajes de la literatura y las distintas situaciones vitales que los textos literarios presentan, ofrecen al estudiante una posibilidad más para integrar a su personalidad, o bien rechazar, nuevos papeles para su desempeño en la vida.

Otro de los objetivos señalados por $M$. consiste en el comentario personal. Al respecto, ella dice: Cuando vimos a Hemingway (se refiere a la novela El viejo y el mar), les bacía 
preguntas como ¿De qué cree usted que hablaba el viejo con el pescado? ¿Qué aprendió usted con el libro?

De nuevo la docente manifiesta una íntima relación entre el tipo de interpretación que ella pide al estudiante y las posibilidades cognoscitivas de este (capacidad para efectuar suposiciones, elaborar hipótesis), así como con sus intereses psicosociales (aplicar lo visto en el texto a su vida, o desecharlo como posible fuente de identificaciones).

\subsubsection{Estrategias de comprensión de lectura literaria}

M. aplica varias estrategias para estimular la comprensión de lectura de textos literarios en sus alumnos: la motivación, el significado personal, el desarrollo de los procesos propios y la contextualización.

\subsubsection{Motivación}

Según M., la motivación debe existir para que se dé el proceso de comprensión de la lectura literaria. Es necesario que el alumno sienta interés por los temas, dice ella.

Una estrategia de motivación que ella emplea consiste en presentar el texto literario a través de una fórmula discursiva conocida y familiar para los alumnos, como la telenovela, el relato de suspenso y hasta los chismes. Les medio cuento un poco a todos del texto a leerse, tipo telenovela mejicana o venezolana, escribe.

Otra forma de motivarlos es el contacto con el medio. Este se logra ya no por el recurso discursivo, sino a través de un acercamiento a algo concreto de lo que trate la obra literaria. Si la novela o los capítulos de la siguiente lección hablan de la naturaleza, me los llevo (a los estudiantes) fuera de la clase...

Observo que en ambas estrategias el interés resulta de lo que es familiar para el alumno: las telenovelas o el medio circundante. Además, el enlace hacia lo que despierte interés se logra mediante los aspectos de contenido del texto (el argumento en el caso del re- curso a la telenovela, y el ambiente espacial en el caso de la salida fuera de la clase). Los elementos formales del texto (tipo de narrador, secuencia lineal o perturbada, figuras literarias, por ejemplo) no suelen, por ellos mismos, dar pie a recursos empleados para motivar.

La motivación enfocada hacia el contenido del texto constituye, según Méndez (1998), una posible aproximación constructivista a los textos literarios, en la medida en que los temas planteados pueden resultat más significativos para los estudiantes.

Un último elemento relacionado con la motivación lo da la pauta de trabajo. La docente no proporciona guías para que los estudiantes analicen los textos. Ellos trabajan sobre lo que más les interese. Según M., ese interés de los alumnos se enfoca siempre hacia los contenidos.

\subsubsection{Significado personal}

Muy relacionada con la motivación, se halla una estrategia de lectura en la cual el estudiante da su significado personal al texto. M. propone que el estudiante lo desarrolle ... a satisfacción personal, que se inmiscuya dentro del texto, que lo haga suyo...

A esta estrategia se une el hecho de que la guía de trabajo la hacen los propios alumnos. De ella se obtienen, según la docente, interpretaciones muy interesantes. Por ejemplo, formulan preguntas tales como ¿De dónde saca Sancho (el personaje de Don Quijote de la Mancha) su sabiduria? respecto de lo cual comenta M: Lo ponen como complemento de don Quijote, no como un subordinado ni un tonto.

Aquí encuentro tres puntos que merecen ser comentados.

En primer lugar, esta estrategia de darle un significado personal al texto se halla en consonancia con los postulados del enfoque transaccional de la comprensión lectora y con los planteamientos de la teoría literaria moderna. Aquella afirma que lector y texto forman parte de una situación total en la que cada uno condiciona y es condicionado por el 
otro, ya que la interpretación que el sujeto dé al texto depende del ambiente cultural al que pertenece (Carrasco, 1999). La teoría literaria más reciente no plantea que el lenguaje literario sea un espejo de la realidad, como lo sostenía Aristóteles en su Poética; todo lo contrario, el texto literario obtiene su significado en una relación dialógica con el lector, relación en la cual texto y lector no constituyen más que prácticas discursivas que acaban confundiéndose como en una banda de Moebius. (Picado, 1985).

En segundo lugar, las diversas interpretaciones que surgen del análisis propio de los estudiantes revelan que la literatura es pluralidad, como señala Barthes, y que de eso justamente se obtiene el placer de leerla. Además, estas interpretaciones de los alumnos a veces contradicen, sin dejar de ser atinadas, la crítica tradicional más ortodoxa, que, en el caso de don Quijote y Sancho Panza, insiste en un señalar una oposición rígida entre ambos personajes.

Por último, tal estrategia resulta acorde con el desarrollo intelectual del adolescente, pues le ofrece posibilidades de estimular su capacidad de abstracción y de elaboración de hipótesis.

\subsubsection{Procesos propios: énfasis en las capacidades analíticas o sintéticas}

M. considera que cada estudiante es un ser integral con todas sus capacidades, pero con diferencias en cuanto a los procesos por los cuales aprende. Todos deben cumplir con ciertos procesos, mas su forma particular de hacerlo variará. Sus expresiones al respecto son: El alumno debe aprender a analizar, a abstraer; a sintetizar y de manera individual. No puede encasillarse a todos los alumnos en lo mismo. Cada uno es una persona con diferentes capacidades. $Y$ en otra parte senala: No pretendo que el alumno sea igual a los demás, ni que aprenda o comprenda igual que los demás, ni por los mismos medios.

Para responder a esta consideración. M. ha desarrollado -"empíricamente"- su propia estrategia al dividir a los alumnos en dos grupos:
- Los analíticos naturales. Con ellos la estrategia de lectura consiste en analizar el texto parte por parte. Ellos presentan dificultades al sintetizar, pero tienen su lado fuerte en la expresión escrita: Son retóricos por excelencia.

- Los sintéticos naturales. Muestran mayor capacidad para el resumen y la abstracción. Con estos alumnos la estrategia que aplica consiste en motivarlos a leer, a partir de lo cual pueden realizar una interpretación sintética al estilo de una fórmula matemática.

Esta estrategia se centra en los estudiantes, especificamente en sus mayores capacidades para el análisis o la síntesis.

\subsubsection{Ubicación contextual}

Una de las principales dificultades señaladas por $M$. para enseñar a leer literatura, consiste en la falta de contextualización de los estudiantes respecto de los problemas o circunstancias que plantean los textos literarios He incluido este aspecto dentro de lo que se considera como "conocimiento previo".

M. utiliza como estrategia la ubicación contextual, que sería, según mi criterio, una forma de dotar, a los estudiantes, de los conocimientos previos que ella juzga necesarios para comprender el texto. Sin contexto no bay comprensión de lectura literaria, afirma la docente.

Este conocimiento previo contempla la ubicación en el contexto cultural de la época. Para M. esto no significa que se les den a los estudiantes datos aislados, como decir simple y llanamente que vallejo era comunista; lo que se debe hacer es explicar las causas, el significado y la valoración del comunismo en la realidad peruana en que vivió Vallejo.

Obsérvese el ejemplo de la mediación que M. efectúa con un poema de Safo: ...cuando tuvieron yue estudiar lírica griega, con la poesia de Safo (...), primeramente les bice todos los chismes de los dioses, de la vida licenciosa 
de Safo, de los enredos dioses-mortales, de la vida licenciosa griega, etc. Casi una novela del asunto. Usted bubiera visto el interés. No podian creer casi el cuento. Por supuesto, se aprendieron la "Oda a Afrodita", más lo relativo a Grecia.

Debo aclarar que los programas del Ministerio de Educación Pública incluyen la ubicación contextual para todos y cada uno de los textos literarios. Pero tal elemento aparece como uno más de una larga lista, es decir, que no se le confiere la importancia que le atribuye $M$.

\subsection{La mediación docente en la ense- ñanza de la literatura}

Hecho este recorrido por las dificultades y las estrategias asociadas a la enseñanza de la literatura que $M$. ha proporcionado con su testimonio, me corresponde ahora señalar algunos puntos relacionados específicamente con la mediación docente, entendida esta en términos amplios.

Según se desprende del caso estudiado, la mediación docente en la lectura literaria se orienta hacia tres objetivos: la valoración de los textos literarios cuya lectura es obligatoria, la valoración de las directrices del Ministerio de Educación Pública en cuanto al análisis literario, y la valoración de los estudiantes

Las estrategias que emplea la docente se centran en los alumnos: en su motivación, su nivel de conocimiento contextual, sus intereses personales. Para ello, la profesora parte de los textos obligatorios, mas no necesariamente del análisis que propone el Ministerio.

La causa de esta situación puede residir en el hecho de que los programas oficiales no se centran en el estudiante, sino en el texto literario mismo. Son elaborados a partir de criterios muy especializados. Por tal motivo, no concuerdan con la propuesta de mediación que efectúa $M$, ya que se centran en aspectos diferentes.

Por otra parte, los programas oficiales están cliseñados desde una postura tradicional cle la comprensión lectora, que privilegia el texto, ante el cual el lector es un ente pasivo. (Debo aclarar que en las sugerencias que anteceden a los contenidos propios del programa, se habla de motivación y de lectura creativa y crítica, pero entre estos criterios y los contenidos no se desarrolla luego una articulación concreta que sustente tales recomendaciones).

\section{Conclusiones}

Seguidamente planteo en forma sucinta los puntos que se desprendieron de la discusión de los resultados. Conviene insistir en que se trata de un estudio de casos y que, por lo tanto, estos resultados no son generalizables.

5.1 Existen dificultades en la enseñanza de la literatura. Estas tienen que ver con los textos, específicamente con la selección de textos literarios hecha en los programas oficiales, la cual se caracteriza por su falta de adaptación al nivel comprensivo de los estudiantes, por su falta de secuencia cronológica y por la incomprensibilidad de algunos textos. También se plantean dificultades en cuanto a la metodología o estrategias de lectura propuestas en los programas oficiales; el análisis literario que en tales documentos se plantea no resulta significativo para el trabajo con los estudiantes. Otra dificultad radica en la formación docente de la profesora, que, según ella, se centra en aspectos formales, sin una articulación con los contenidos propios de lá disciplina literaria. Asimismo, la actitud de los lectores plantea una dificultad: los estudiantes de enseñanza media se muestran desinteresados hacia la lectura de unos textos literarios cuyo carácter es obligatorio, y respecto de los cuales carecen del conocimiento previo necesario para su comprensión. Por último, el carácter no normativo de la literatura la convierte en una disciplina cuya enseñanza se torna mucho más difícil que, por ejemplo, la de la gramática.

5.2 La comprensión de lectura de textos literarios desde la mediación docente parte de un concepto de lectura literaria en términos de desarrollo de lá creatividad, formación de un 
pensamiento abstracto y logro de una formación integral. Las estrategias asociadas con este concepto enfatizan en la motivación, la ubicación contextual y el reconocimiento de la individualidad de cada estudiante. La docente desarrolla la motivación empleando estrategias como la presentación del texto literario a través de una fórmula discursiva conocida y familiar para los alumnos, y también por el contacto con el medio que los rodea; asimismo, aplicando una pauta de trabajo personal; la estrategia de darle un significado personal al texto está en consonancia con la teoría transaccional de la comprensión lectora, y la pluralidad de significados resultante de esta actividad pone en evidencia esta característica de los textos literarios. Para enfatizar en los procesos propios de acuerdo con las distintas capacidades de los estudiantes, la docente ha desarrollado una clasificación: los analíticos naturales y los sintéticos naturales. Por último, la ubicación contextual desarrollada por $M$. consiste en dotar al estudiante del conocimiento previo necesario para una mayor comprensión del texto literario.

5.3 A manera de sintesis, la mediación docente en la enseñanza de la literatura abarca tres puntos: la valoración de los textos literarios de lectura obligatoria; la valoración de las directrices que, en materia de análisis literario, se desprenden de los programas del Ministerio de Educación Pública; y la consideración de las habilidades e intereses de los estudiantes. La docente actúa, de esta forma, como una especie de intermediario entre unas directrices programáticas (que parecen privilegiar el texto literario por sobre las necesidades, gustos e intereses de los estudiantes, a quienes se obliga a leer determinadas obras) y sus grupos de alumnos (que manifiestan actitudes de rechazo hacia unos textos literarios que no comprenden, pues ellos carecen de los conocimientos previos necesarios para interpretar los diversos elementos configuradores de tales obras literarias).

\section{Referencias Bibliogräficas}

Bendito, Ana T. de. "¿Se aprende a leer escribiendo?". En: Lectura y vida. Revis- ta latinoamericana de lectura, año 10 , número 4, pp. 12-17. 1989.

Borges, Jorge Luis. "La poesía". En: Siete noches. México: Fondo de Cultura Económica, 1988.

Carrasco Altamirano, Alma. "La lectura de textos narrativos entre estudiantes de los últimos grados de primaria en México". En: Lectura y vida. Revista latinoamericana de lectura, año 20 , número 1, pp. 15-20, 1999.

Costa Rica, Ministerio de Educación Pública. Español: tercer ciclo. San José: El Ministerio, 1995.

Craig, Grace J. Desarrollo psicológico. México: Prentice-Hall Hispanoamericana, sétima edición, 1997.

Dengo, María Eugenia. "Desarrollo de la formación docente en Costa Rica". En: Salazar, Jorge Mario (coordinador). Educación costarricense. San José: EU$\mathrm{NED}$, en prensa.

Dobles Izaguirre, María Cecilia; Zúñiga Céspedes, Magaly; García Fallas, Jackeline. Investigación en educación: procesos, interacciones, construcciones. San José: EUNED, 1996.

Ducca, Isabel y Rojas, Marta. Despertando a las palabras: libro de lectura. cuarto grado. San José: ECR, 1993.

Dubois, María Eugenia. "Lectura, escritura y formación docente". En: Lectura y vida. Revista latinoamericana de lectura, año 16 , número 2 , pp. 5-11, 1995.

Krauskopf, Dina. Adolescencia y educación. San José: EUNED, octava reimpresión de la primera edición, 1993.

La Nación. "MEP rechaza baja en cobertura". 17 de julio de 1999, pág. 16 A. 
Méndez Anchía, Silvia. Tratamientos didáctico, temático, axiológico, formal y teórico-literario en Textos de lectura y comentarios para noveno año. Informe final de curso especializado para optar al grado de Licenciatura en Docencia, Universidad Estatal a Distancia, 1998.

Piaget, Jean. Seis estudios de psicologia. Barcelona: Editorial Ariel, 1983.

Picado, Manuel. El envés de la red. San José: EDUCA, 1985.

Pujol, Lidia y Vivas, Eleonora. "Prevalencia de desempeño en lectura en escolares venezolanos: dificultades según tipo de texto". En: Lectura y vida. Revista latinoamericana de lectura, año 18 , número 1, pp. 17-26, 1997.

Riffo Ocares, Bernardo. "Niveles de procesamiento en la comprensión del discurso narrativo". En: lectura y vida. Revista latinoamericana de lectura, año 18, número 1, pp. 5-16, 1997.

Roclino Pierri, Ana María y Ross, L. Ronald. Problemas de expresion escrita del estudiante universitario costarricense. San José: EUNED, segunda reimpresión de la primera edición, 1997.

Rojas Porras, Marta; Viquez Jiménez, Alí; Sánchez Corrales, Víctor. Español 8: texto. San José: EUCR, 1996.

Rojas Salazar, María Emilce. "Entrevista sobre estrategials de comprensión lectora" realizada por Silvia Méndez Anchía el 1 de junio cle 1999.

Sierra Bravo, Restituto. Técnicas de investigación social. Teoria y ejercicios. Madrid: Editorial Paraninfo, 1994.

Solé, Isabel. "Estrategias de comprensión de la lectura". En: Lectura y vida. Revista latinoamericana de lectura, año 17 , número 4, pP. 5-22, 1996

Stake, R. E. Investigación con estudio de casos. Madrid: Ediciones Morata, 1998.

Taylor, S. J. y Bogdan, R. Introducción a los métodos chalitativos de investigación. La buisqueda de significados. Barcelona: Edicionnes pádicós. 1992. 